\title{
IR diagnostics of embedded jets: kinematics and physical characteristics of the HH46-47 jet ${ }^{\star}$
}

\author{
R. Garcia Lopez ${ }^{1}$, B. Nisini ${ }^{1}$, J. Eislöffel ${ }^{2}$, T. Giannini ${ }^{1}$, F. Bacciotti ${ }^{3}$, and L. Podio ${ }^{4}$ \\ 1 INAF-Osservatorio Astronomico di Roma, via di Frascati 33, 00040 Monteporzio Catone, Italy \\ e-mail: garcia@oa-roma.inaf.it \\ 2 Thüringer Landessternwarte Tautenburg, Sternwarte 5, 07778 Tautenburg, Germany \\ 3 INAF-Osservatorio Astrofisico di Arcetri, Largo E. Fermi 5, 50125 Florence, Italy \\ 4 Dublin Institute for Advanced Studies, 31 Fitzwilliam Place, Dublin 2, Ireland
}

Received 16 September 2009 / Accepted 6 December 2009

\section{ABSTRACT}

\begin{abstract}
Context. We present an analysis of the kinematics and physical properties of the Class I driven jet HH46-47 based on IR medium and low resolution spectroscopy obtained with ISAAC on VLT.

Aims. Our aim is to study the gas physics as a function of the velocity and distance from the source and to compare the results with similar studies performed on other Class I and classical T Tauri jets as well as with existing models for the jet formation and excitation. Methods. The ratios and luminosities of several important diagnostic lines (e.g. [Fe II] 1.644, $1.600 \mu \mathrm{m}$, [P II] $1.189 \mu \mathrm{m}$, and $\mathrm{H}_{2}$ lines) have been used to derive physical parameters such as electron density, $\mathrm{H}_{2}$ temperature, iron gas-phase abundance and mass flux. [Fe II] $1.644 \mu \mathrm{m}$ and $\mathrm{H}_{2} 2.122 \mu \mathrm{m}$ position velocity diagrams (PVDs) have been additionally constructed to study the kinematics of both the atomic and molecular gas.

Results. Within 1000-2000 AU from the source the atomic gas presents a wide range of radial velocities, from $\sim-230 \mathrm{~km} \mathrm{~s}^{-1}$ to $\sim 100 \mathrm{~km} \mathrm{~s}^{-1}$. Only the gas component at the highest velocity (high velocity component, HVC) survives at large distances. The $\mathrm{H}_{2}$ shows only a single velocity component at almost zero velocity close to the source while it reaches higer velocities (up to $\sim 95 \mathrm{~km} \mathrm{~s}^{-1}$ ) further downstream. Electron densities $\left(n_{\mathrm{e}}\right)$ and mass ejection fluxes $\left(\dot{M}_{\text {jet }}\right)$ have been separately measured for the HVC and for the component at lower velocity (LVC) from the [Fe II] lines. $n_{\mathrm{e}}$ increases with decreasing velocities with an average value of $\sim 6000 \mathrm{~cm}^{-3}$ for the LVC and $\sim 4000 \mathrm{~cm}^{-3}$ for the HVC, while the opposite occurs for $\dot{M}_{\text {jet }}$ which is $\sim 0.5-2 \times 10^{-7} M_{\odot} \mathrm{yr}^{-1}$ and $\sim 0.5-3.6 \times$ $10^{-8} M_{\odot} \mathrm{yr}^{-1}$ for the HVC and LVC, respectively. The mass flux carried out by the molecular component, measured from the $\mathrm{H}_{2}$ lines flux, is $\sim 4 \times 10^{-9} M_{\odot} \mathrm{yr}^{-1}$. We have estimated that the $\mathrm{Fe}$ gas phase abundance is significantly lower than the solar value, with $\sim 88 \%$ of iron still depleted onto dust grains in the internal jet region. This fraction decreases to $\sim 58 \%$, in the external knots.

Conclusions. Many of the derived properties of the HH46-47 jet are common to jets from young stellar objects (YSOs) in different evolutionary states. The derived densities and mass flux values are typical of Class I objects or very active T Tauri stars. However, the spatial extent of the LVC and the velocity dependence of the electron density have been so far observed only in another Class I jet, the HH34 jet, and are not explained by the current models of jet launching.
\end{abstract}

Key words. circumstellar matter - Herbig-Haro objects - ISM: jets and outflows - ISM: individual objects: HH46-47 - infrared: ISM

\section{Introduction}

Protostellar jets are tightly associated with the first stage of protostar evolution. They are believed to remove angular momentum, disrupt infalling material from the cloud, inject turbulence in the ISM and modify its chemistry. Little is known, however, about the origin of protostellar jets. There are two principal schools of thought regarding where protostellar jets are launched from: the X-wind and the disc-wind models (Shu et al. 1995; Ferreira 1997). While disc-wind models assume ejection from a large spread of radii from the disc, $\mathrm{X}$-wind models assume that the ejection of material takes place only at a single annulus at the interaction region between the stellar magnetosphere and the inner edge of the disc. Both models were born in an attempt to explain the kinematical and physical properties observed at optical wavelengths in jets from classical T Tauri stars (e.g., Bacciotti et al. 2000; Lavalley et al. 1997). Very few studies have been done though regarding the physical properties of Class I jets.

* Based on observations collected at the European Southern Observatory, Paranal, Chile (ESO programmes 0.74.C-0286(A)).
Since these objects are still highly embedded in their molecular clouds, they are affected by a large extinction. Thus it is not possible to detect the region near to the exciting source at optical wavelengths. In this context, infrared spectroscopy is a very useful tool to probe the physical properties and kinematics of Class I jets close to their base, where their characteristics have not yet been modified by the interaction with the surrounding material. Several studies have been carried out in recent years employing near-IR (NIR) line diagnostic on protostellar jets (e.g., Nisini et al. 2005; Podio et al. 2006; Davis et al. 2006; Takami et al. 2006). The main lines used to probe physical and kinematical properties in NIR are [Fe II] (e.g. $1.644 \mu \mathrm{m}, 1.600 \mu \mathrm{m}$ ) and $\mathrm{H}_{2}$ lines (e.g. $2.122 \mu \mathrm{m}$ ). These studies revealed that the kinematical structure at the jet base of Class I and classical T Tauri stars (CTTSs) jets is very similar. Both show a broad line emission near the source which consists of a collimated and large scale jet at high velocity (the so-called HVC) and a slower velocity component (the so-called LVC) that is only detected around the central source. Noticeably, this kind of velocity structure is often observed in both the atomic and molecular components of 
the jets, the latter probed through the $\mathrm{H}_{2}$ emission and called molecular hydrogen emission line regions (MHEL; Davis et al. 2001b). Concerning the physical properties, Class I jets show higher densities and mass loss rates than CTTS jets, as expected for less evolved objects. Both disc-wind and X-wind models predict the presence of a large spread of velocity at the jet base as shown by the few synthetic position velocity diagrams (PVDs) constructed for both models (Shang et al. 1998; Pesenti et al. 2004), although attempts to reproduce the specific characteristics (e.g. kinematics, presence of the MHEL region, physical parameters) displayed by the Class I jets are still lacking. We have now started a project aimed to determine a few basic physical parameters of embedded jets as a function of both velocity and distance from the driving source, using near-IR (NIR) medium resolution $(R \sim 10000)$ spectra taken with ISAAC on the Very Large Telescope (VLT). The goals of this study are to make a quantitative comparison between the physical properties of Class I and CTTS jets and to constrain the origin of the different velocity components and the mechanism responsible for their excitation. In a previous paper (Garcia Lopez et al. 2008), we have applied our NIR diagnostic techniques to two jets, HH34 and HH1.

We now extend this previous study to the HH46-47 jet. This known Class I jet is at a distance of $\sim 450 \mathrm{pc}$ emerging from a dense reflecting nebula. The jet is located at the border of a Bok globule and powers an atomic and molecular bipolar flow. The driving source (HH46-IRS) is in a phase of high accretion (Antoniucci et al. 2008) and is known to be a deeply embedded binary system (Reipurth et al. 2000). The northeastern (blueshifted) portion of the flow is detected at optical wavelengths giving rise to strong $\mathrm{HH}$ objects (Hartigan et al. 1993, 2005). Recently, the kinematics of the internal region of this optical jet have been studied in detail by Nishikawa et al. (2008). At infrared wavelengths, the HH46 jet and its red-shifted counter-jet are detected down to the central source (Eislöffel et al. 1994), thus this object represents a very suitable target for our NIR spectroscopy study.

In Sect. 2 we present the observations and the data reduction. In Sect. 3 we describe the results of our observations, while the kinematics of the atomic and molecular components are presented in Sect. 4. In Sects. 5 and 6 we describe the spectral diagnostic analysis applied to ISAAC spectra obtained at low and medium resolution, respectively. We have compared the results found on the HH46-47 jet with the HH34 jet in Sect. 7, and the main conclusions of our work are finally summarised in Sect. 8 .

\section{Observations and data reduction}

The observations were made on 29-30 December 2004 at the ESO VLT telescope on Cerro Paranal, Chile, using the infrared spectrograph and camera ISAAC. The spatial scale of the instrument is 0 '. $^{\prime} 148 /$ pixel. We have taken low resolution spectra in the $J$-band $(1.1-1.4 \mu \mathrm{m})$ and both medium and low resolution spectra in the $H(1.42-1.82 \mu \mathrm{m})$ and $K(1.82-2.5 \mu \mathrm{m})$ bands. The adopted slit widths were $0 . ' 6$ and $0 . ' 3$ for the low and medium resolution, respectively, corresponding to nominal resolutions of 860 (in $J$ ), 840 (in $H$ ) and 750 (in $K$ ) at low resolution (LR) and 10000 (in $H$ ) and 8900 (in $K$ ) at medium resolution (MR). The slits were all aligned along the jet, with a position angle (PA) of $\sim 57^{\circ}$. Total integration times were $600 \mathrm{~s}$ for each filter in the LR mode and $3600 \mathrm{~s}$ and $7200 \mathrm{~s}$ for the $K$ - and $H$-band in the MR mode.

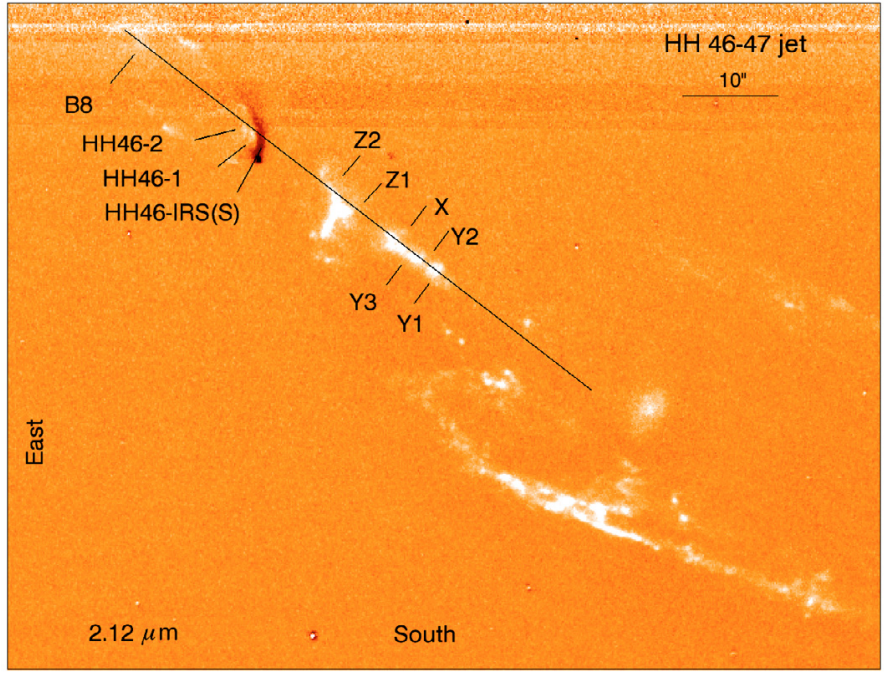

Fig. 1. Position of the ISAAC slit superimposed on the $\mathrm{H}_{2} 2.12 \mu \mathrm{m}$ image of the HH46-47 jet. At the source (HH46-IRS) and knot S position a negative residual from the continuum substraction is present. Individual knots along the jet are identified following and our own (see text) nomenclature and that of Eislöffel \& Mundt (1994). The northeastern knots correspond to the blue-shifted jet.

Data reduction was performed using standard IRAF $^{1}$ tasks. Wavelength calibration was done using the atmospheric $\mathrm{OH}$ emission at MR and an argon lamp at LR. The atmospheric spectral response was corrected by dividing the object spectra by the spectrum of a telluric standard star for both modes. Flux calibrations were performed using photometric standards observed at an air mass and seeing similar to our target observations. This procedure probably leads to some flux losses due to the finite slit width with respect to the jet width, especially in the case of the bow shaped knot Z1. In MR spectroscopy, the continuum of the HH46-IRS source was subtracted using the IRAF task BACKGROUND.

The reduced MR spectra on the source position have been already presented in Antoniucci et al. (2008) (reduced and analysed in the same way as explained above) and thus they will not be discussed in the present paper.

\section{Results}

Figure 1 shows an $\mathrm{H}_{2} 2.12 \mu \mathrm{m}$ continuum-subtracted image of the HH46-47 jet. The slit position is depicted over the image and the covered knots are named following the nomenclature of Eislöffel \& Mundt (1994). In addition, we name as knot $\mathrm{S}$ the emission that peaks at the source position and extends for $\sim 3^{\prime \prime}$ in the blue-shifted jet. The observations cover the blue-shifted jet knots HH46-1 and 2 and the knot B8, which is located at the apex of a bow shock. In the red-shifted lobe, the slit comprises knots Z and Y in Eislöffel \& Mundt (1994) that have been separated here in knots $\mathrm{Z} 1$ and $\mathrm{Z} 2$ and $\mathrm{Y} 1, \mathrm{Y} 2$ and $\mathrm{Y} 3$.

The low resolution spectra present several lines of both atomic and molecular species in the different knots. Figure 2 shows the complete spectrum of knot $\mathrm{Z1}$, which is among the brightest ones along the jet. A list of the observed lines, fluxes and identification in knot $\mathrm{Z} 1$ is also reported in Table 1, while

\footnotetext{
1 IRAF (Image Reduction and Analysis Facility) is distributed by the National Optical Astronomy Observatories, which are operated by AURA, Inc., cooperative agreement with the National Science Foundation.
} 


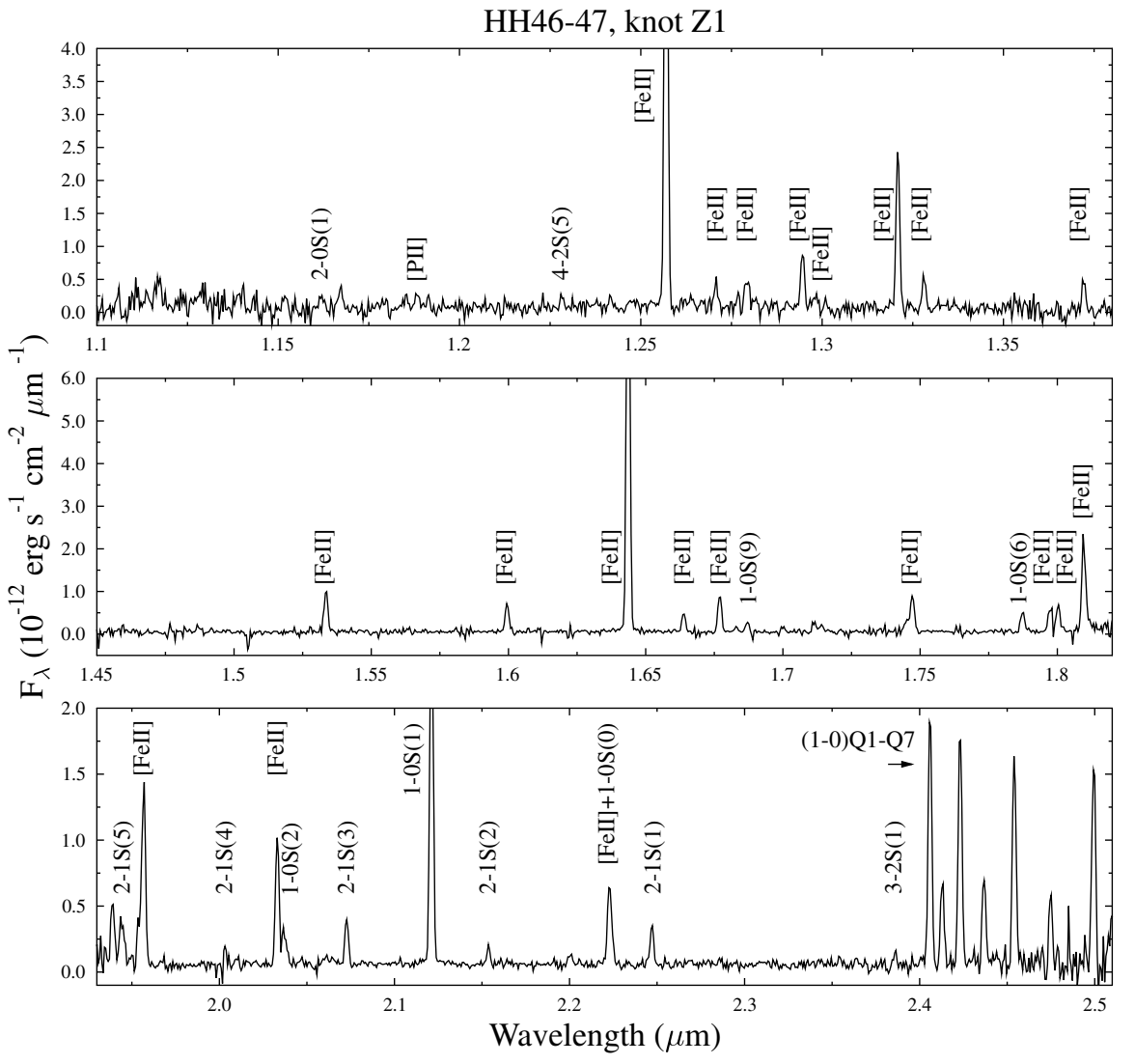

Fig. 2. LR spectrum from 1.00 to $2.50 \mu \mathrm{m}$ of the knot $\mathrm{Z} 1$ in the HH46-47 jet. The strongest lines are identified.
Tables A. 1 and A. 2 in the Appendix A list all the lines detected in the other knots. [Fe II] transitions from the first 12 fine-structure levels and $\mathrm{H}_{2}$ transitions from the two first vibrational levels have been detected in all of the knots at various intensity levels. The knot $\mathrm{S}$ is together with the knot $\mathrm{Z} 1$ the richest in ionic lines. Here $[\mathrm{P} \mathrm{II}]{ }^{3} \mathrm{P}_{2}-{ }^{1} \mathrm{D}_{2} 1.189 \mu$ mhas also been detected.

The medium resolution $K$-band spectra show several lines, the brightest being $\mathrm{H}_{2} 1-0 \mathrm{~S}(1), 3-2 \mathrm{~S}(4)$ and $2-1 \mathrm{~S}(2)$, [Fe II] 2.1349 and $2.1609 \mu \mathrm{m}$ and $\mathrm{Br} \gamma$ (see, Fig. 3). In addition, the [Fe II] 1.600 and $1.644 \mu \mathrm{m}$ lines have been detected in the $\mathrm{H}$-band spectra. The presence of the $\mathrm{Br} \gamma$ near the source and the $\mathrm{H}_{2} 3-2 \mathrm{~S}(4)$ line at a distance of $10^{\prime \prime}$ to $25^{\prime \prime}$ from HH46-IRS testifies to the high excitation conditions in these regions. In addition, the on-source spectrum shows other features, as the Br14 and $\mathrm{Br} 12$ and $\mathrm{CO}$ transitions (see also, Antoniucci et al. 2008).

\section{Kinematics}

In order to study the kinematics of the atomic and molecular component of the HH46-47 jet as a function of distance from the central source, we have constructed PVDs of the [Fe II] $1.644 \mu \mathrm{m}$ and $\mathrm{H}_{2} 2.122 \mu \mathrm{m}$ lines (see Fig. 5) from the MR spectral images. The velocity has been expressed with respect to the local standard of rest (LSR) and corrected for a parental cloud velocity of $+20 \mathrm{~km} \mathrm{~s}^{-1}$ (Hartigan et al. 1993). In the $Y$-axis the distance from the driving source HH46-IRS is represented in arcsec. The continuum of the source has been subtracted in both the PVDs to see the structure of the lines near HH46-IRS more clearly. The intensity scale is different in the two PVDs to show the different condensations in the atomic and molecular gas.

Emission down to the central source is detected for both the atomic and molecular components $(\mathrm{knot} \mathrm{S})$. The [Fe II] emission broades as it approaches the source position and spans velocities from $\sim-200 \mathrm{~km} \mathrm{~s}^{-1}$ down to $0 \mathrm{~km} \mathrm{~s}^{-1}$ within $2^{\prime \prime}$ from the source. Inside $1^{\prime \prime}$ there is also emission at red-shifted velocities up to $\sim+100 \mathrm{~km} \mathrm{~s}^{-1}$. We will call the blue-shifted component at high velocity, which can be traced up to large scale the high velocity component (HVC) and the more compact emission component from $\sim-150 \mathrm{~km} \mathrm{~s}^{-1}$ to $\sim 0 \mathrm{~km} \mathrm{~s}^{-1}$ the low velocity component (LVC). These two components have been also evidenced in $\mathrm{H} \alpha$ optical observations (Nishikawa et al. 2008). At variance with the ionic emission, the $\mathrm{H}_{2}$ emission close to the source (knot S) shows only one component at nearly $0 \mathrm{~km} \mathrm{~s}^{-1}$.

The radial velocities of the $[\mathrm{Fe}$ II $] 1.644 \mu \mathrm{m}$ and $\mathrm{H}_{2} 2.122 \mu \mathrm{m}$ lines are reported in Table 2. They have been computed by a Gaussian fit to the line profile of every knot. The velocities of the $\left[\mathrm{Fe}\right.$ II] emission range from $-235 \mathrm{~km} \mathrm{~s}^{-1}$ to $-177 \mathrm{~km} \mathrm{~s}^{-1}$ and from $+144 \mathrm{~km} \mathrm{~s}^{-1}$ to $+94 \mathrm{~km} \mathrm{~s}^{-1}$ in the blueand red-lobes, respectively: thus in both lobes the peak velocity decreases as the distance from the driving source increases in both lobes. The HVC and LVC in knot S peak at $-213 \mathrm{~km} \mathrm{~s}^{-1}$ and around $-138 \mathrm{~km} \mathrm{~s}^{-1}$. At knot $\mathrm{Z} 2$ the emission splits into two separate velocity components (at $+144 \mathrm{~km} \mathrm{~s}^{-1}$ and $+92 \mathrm{~km} \mathrm{~s}^{-1}$ ) whose origin is likely connected with the presence of the large bow shock structure.

The radial velocities of the $\mathrm{H}_{2}$ emission vary from $-15 \mathrm{~km} \mathrm{~s}^{-1}$ to $-48 \mathrm{~km} \mathrm{~s}^{-1}$ in the blue-lobe and from $+26 \mathrm{~km} \mathrm{~s}^{-1}$ to $+94 \mathrm{~km} \mathrm{~s}^{-1}$ in the red-lobe. At variance with the [Fe II] emission, knot $\mathrm{S}$ only shows a single velocity component at $15 \mathrm{~km} \mathrm{~s}^{-1}$, while both knots $\mathrm{Z} 1$ and $\mathrm{X}$ present two velocity components at $\sim+90 \mathrm{~km} \mathrm{~s}^{-1}$ and $+10 \mathrm{~km} \mathrm{~s}^{-1}$. The high and low velocity components present in these knots and the broad profiles seen in $\mathrm{H}_{2}$ (see, Fig. 4) are similar to the profiles observed in other molecular jets (e.g., Davis et al. 2001a). As shown in Fig. 4, the relative contribution of the high and low velocities components to the total flux changes from knot Z1 (where the 
Table 1. Lines observed in the knot $\mathrm{Z1}$ of the HH46-47 jet.

\begin{tabular}{|c|c|c|c|c|c|c|c|}
\hline Line id. & $\begin{array}{c}\lambda \\
(\mu \mathrm{m})\end{array}$ & $\begin{array}{c}F \\
\left(10^{-16}\right. \\
\end{array}$ & 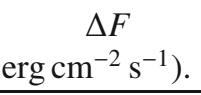 & Line id. & $\begin{array}{c}\lambda \\
(\mu \mathrm{m})\end{array}$ & $\begin{array}{c}F \\
\left(10^{-16}\right. \\
\end{array}$ & 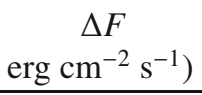 \\
\hline$[\mathrm{Ti}$ II $]{ }^{2} \mathrm{G}_{7 / 2}-{ }^{4} \mathrm{~F}_{7 / 2}$ & 1.140 & 8.3 & 3.5 & $\mathrm{H}_{2} 1-0 \mathrm{~S}(6)$ & 1.788 & 9.4 & 0.5 \\
\hline $\mathrm{H}_{2} 2-0 \mathrm{~S}(1)$ & 1.162 & 5.8 & 2.2 & {$[\mathrm{Fe}$ II $] \mathrm{a}^{4} \mathrm{D}_{3 / 2}-\mathrm{a}^{4} \mathrm{~F}_{3 / 2}$} & 1.797 & 9.8 & 1.1 \\
\hline$[\mathrm{P} I \mathrm{II}]{ }^{3} \mathrm{P}_{2}-{ }^{1} \mathrm{D}_{2}$ & 1.188 & 3.5 & 1.0 & {$[\mathrm{Fe}$ II $] \mathrm{a}^{4} \mathrm{D}_{5 / 2}-\mathrm{a}^{4} \mathrm{~F}_{5 / 2}$} & 1.800 & 7.1 & 0.8 \\
\hline$[\mathrm{Fe}$ II $] \mathrm{a}^{4} \mathrm{D}_{7 / 2}-\mathrm{a}^{6} \mathrm{D}_{9 / 2}$ & 1.257 & 106.0 & 0.8 & {$[\mathrm{Fe}$ II $] \mathrm{a}^{4} \mathrm{D}_{7 / 2}-\mathrm{a}^{4} \mathrm{~F}_{7 / 2}$} & 1.810 & 38.2 & 2.1 \\
\hline$[\mathrm{Fe} I \mathrm{II}] \mathrm{a}^{4} \mathrm{D}_{1 / 2}-\mathrm{a}^{6} \mathrm{D}_{1 / 2}$ & 1.271 & 5.9 & 1.0 & $\mathrm{H}_{2} 2-1 \mathrm{~S}(5)$ & 1.945 & 11.0 & 1.5 \\
\hline$[\mathrm{Fe}$ II $] \mathrm{a}^{4} \mathrm{D}_{3 / 2}-\mathrm{a}^{6} \mathrm{D}_{3 / 2}$ & 1.279 & 7.8 & 0.1 & $\mathrm{H}_{2} 2-1 \mathrm{~S}(4)$ & 2.004 & 2.7 & 0.3 \\
\hline$[\mathrm{Fe}$ II $] \mathrm{a}^{4} \mathrm{D}_{5 / 2}-\mathrm{a}^{6} \mathrm{D}_{5 / 2}$ & 1.295 & 11.9 & 0.9 & $\mathrm{H}_{2} 1-0 \mathrm{~S}(2)$ & 2.034 & 26.1 & 0.6 \\
\hline$[\mathrm{Fe}$ II $] \mathrm{a}^{4} \mathrm{D}_{3 / 2}-\mathrm{a}^{6} \mathrm{D}_{1 / 2}$ & 1.298 & 6.7 & 0.1 & $\mathrm{H}_{2} 2-1 \mathrm{~S}(3)$ & 2.074 & 8.1 & 0.5 \\
\hline$[\mathrm{Fe}$ II $] \mathrm{a}^{4} \mathrm{D}_{7 / 2}-\mathrm{a}^{6} \mathrm{D}_{7 / 2}$ & 1.321 & 306.0 & 0.8 & $\mathrm{H}_{2} 1-0 \mathrm{~S}(1)$ & 2.122 & 68.5 & 0.3 \\
\hline$[\mathrm{Fe}$ II $] \mathrm{a}^{4} \mathrm{D}_{5 / 2}-\mathrm{a}^{6} \mathrm{D}_{3 / 2}$ & 1.328 & 8.4 & 0.9 & $\mathrm{H}_{2} 2-1 \mathrm{~S}(2)$ & 2.154 & 3.9 & 0.3 \\
\hline$[\mathrm{Fe}$ II $] \mathrm{a}^{4} \mathrm{D}_{7 / 2}-\mathrm{a}^{6} \mathrm{D}_{5 / 2}$ & 1.372 & 71.7 & 1.0 & $\mathrm{H}_{2} 2-1 \mathrm{~S}(1)$ & 2.248 & 8.5 & 0.5 \\
\hline$[\mathrm{Fe}$ II $] \mathrm{a}^{4} \mathrm{D}_{5 / 2}-\mathrm{a}^{4} \mathrm{~F}_{9 / 2}$ & 1.534 & 173.0 & 0.7 & $\mathrm{H}_{2} 3-2 \mathrm{~S}(1)$ & 2.386 & 1.6 & 0.4 \\
\hline$[\mathrm{Fe}$ II $] \mathrm{a}^{4} \mathrm{D}_{3 / 2}-\mathrm{a}^{4} \mathrm{~F}_{7 / 2}$ & 1.600 & 10.6 & 0.5 & $\mathrm{H}_{2} 1-0 \mathrm{Q}(1)$ & 2.407 & 50.0 & 0.8 \\
\hline$[\mathrm{Fe}$ II $] \mathrm{a}^{4} \mathrm{D}_{7 / 2}-\mathrm{a}^{4} \mathrm{~F}_{9 / 2}$ & 1.644 & 160.0 & 0.6 & $\mathrm{H}_{2} 1-0 \mathrm{Q}(2)$ & 2.413 & 18.3 & 0.9 \\
\hline$[\mathrm{Fe}$ II $] \mathrm{a}^{4} \mathrm{D}_{1 / 2}-\mathrm{a}^{4} \mathrm{~F}_{5 / 2}$ & 1.664 & 7.0 & 0.7 & $\mathrm{H}_{2} 1-0 \mathrm{Q}(3)$ & 2.424 & 47.4 & 0.9 \\
\hline$[\mathrm{Fe}$ II $] \mathrm{a}^{4} \mathrm{D}_{5 / 2}-\mathrm{a}^{4} \mathrm{~F}_{7 / 2}$ & 1.677 & 142.0 & 0.3 & $\mathrm{H}_{2} 1-0 \mathrm{Q}(4)$ & 2.437 & 18.9 & 1.3 \\
\hline $\mathrm{H}_{2} 1-0 \mathrm{~S}(9)$ & 1.687 & 4.2 & 0.5 & $\mathrm{H}_{2} 1-0 \mathrm{Q}(5)$ & 2.455 & 42.9 & 1.5 \\
\hline$[\mathrm{Fe}$ II $] \mathrm{a}^{4} \mathrm{D}_{3 / 2}-\mathrm{a}^{4} \mathrm{~F}_{5 / 2}$ & 1.712 & 2.1 & 0.4 & $\mathrm{H}_{2} 1-0 \mathrm{Q}(5)$ & 2.455 & 42.9 & 1.5 \\
\hline$[\mathrm{Fe} I \mathrm{II}] \mathrm{a}^{4} \mathrm{D}_{1 / 2}-\mathrm{a}^{4} \mathrm{~F}_{3 / 2} 2 *$ & 1.745 & 6.3 & 0.8 & $\mathrm{H}_{2} 1-0 \mathrm{Q}(7)$ & 2.500 & 47.4 & 3.7 \\
\hline $\mathrm{H}_{2} 1-0 \mathrm{~S}(7)^{3 *}$ & 1.748 & 150.0 & 0.6 & & & & \\
\hline
\end{tabular}

Notes. ${ }^{(*)}$ Blend of the two indicated lines.

Table 2. Observed radial velocities along the HH46-47 jet.

\begin{tabular}{ccc}
\hline \hline Knot & $\begin{array}{c}{[\mathrm{Fe} \mathrm{II}] 1.644 \mu \mathrm{m}} \\
v\left(\mathrm{~km} \mathrm{~s}^{-1}\right)\end{array}$ & $\begin{array}{c}\mathrm{H}_{2} 2.122 \mu \mathrm{m} \\
v\left(\mathrm{~km} \mathrm{~s}^{-1}\right)\end{array}$ \\
\hline B8 & -177 & \\
HH46-1 & -235 & -20 \\
$\mathrm{~S}$ & $-213,(-138)$ & -15 \\
$\mathrm{Z} 2$ & $+144,+92$ & +26 \\
$\mathrm{Z} 1$ & +112 & $+89,+10$ \\
$\mathrm{X}$ & +94 & $+93,+14$ \\
$\mathrm{Y} 3$ & & +94 \\
$\mathrm{Y} 2$ & & +92 \\
$\mathrm{Y} 1$ & & +92 \\
\hline
\end{tabular}

Notes. Radial velocities corrected from a cloud velocity of $+20 \mathrm{~km} \mathrm{~s}^{-1}$ (Hartigan et al. 1993) and with respect to the LSR. The radial velocity error is $2 \mathrm{~km} \mathrm{~s}^{-1}$.

component at $0 \mathrm{~km} \mathrm{~s}^{-1}$ is larger than that at high velocity) to knot $\mathrm{X}$ (where the component at $0 \mathrm{~km} \mathrm{~s}^{-1}$ is significantly reduced). These profiles are similar to those expected from bowshock structures viewed at a certain inclination angle (Schultz et al. 2005). With respect to the Schultz et al. (2005) models, the HVC and LVC contribution to the total flux depend in our case not only on the viewing angle of the bow-shock, but also on how the $\mathrm{H}_{2}$ cooling is distributed from the apex to the wings of the bow. In particular the profiles of the knots $\mathrm{Z} 1$ and $\mathrm{X}$ are consistent with bow-shocks seen at $30^{\circ}$ with respect to the line of sight, but with a different relative contribution to the $\mathrm{H}_{2}$ cooling between the apex and the wing. In knot $X$ the apex mostly contributes to the total flux, while in knot $\mathrm{Z} 1$ the situation is the opposite. Such an effect could be actually due to a poor alignment of the slit with the bow-shock apex in knot Z1.

\section{Low resolution spectral analysis}

\subsection{Extinction}

Visual extinction values can be derived from the [Fe II] $1.644 / 1.257 \mu \mathrm{m}$ ratio: these lines share the same upper level, and thus their theoretical ratio is a function of the frequencies and probabilities of the transition and is independent of the plasma conditions. To convert the observed ratios into $A_{v}$ values, we have adopted the radiative transition probabilities given by Quinet et al. (1996) using the relativistic Hartree-Fock (HFR) computer programme. The extinction errors have been estimated directly from the flux error of each line. The results are reported in Table 3 for the individual knots where the $\mathrm{S} / \mathrm{N}$ of the two lines is larger than 10 .

$A_{V}$ varies from $\sim 6$ in the knots $\mathrm{Z} 1$ and $\mathrm{S}$ to $\sim 12$ in the knot Z2. Fernandes (2000) found a value of $A_{V}=9.38 \pm$ $1.49 \mathrm{mag}$ in a region that covers our knots HH46-1 and S. This value has however been derived employing the theoretical [Fe II] $1.644 / 1.257 \mu \mathrm{m}$ ratio given by the radiative rates of Nussbaumer \& Storey (1988) that predict higher values with respect to the Quinet et al. (1996) rates (Nisini et al. 2005). On the other hand, Antoniucci et al. (2008) found an $A_{V} \sim 40 \mathrm{mag}$ on-source, indicating a high density gradient towards the central object. One should note here that the extinction found by Antoniucci et al. (2008) on the basis of photometric measurements coupled with $\operatorname{Br} \gamma$ emission is higher than the one we found on the knot $\mathrm{S}$ since [Fe II] lines trace more external regions. In addition, the knot $\mathrm{S}$ covers a region that extends $\sim 3^{\prime \prime}$ around the source position.

The extinction in the knot $\mathrm{Z} 2$ is roughly twice the extinction of the knots $\mathrm{S}$ and $\mathrm{Z1}$. This fact could be due to the collision of the jet with stationary material on a side of the cavity. Heathcote et al. (1996) note the presence of an oblique shock due to the 


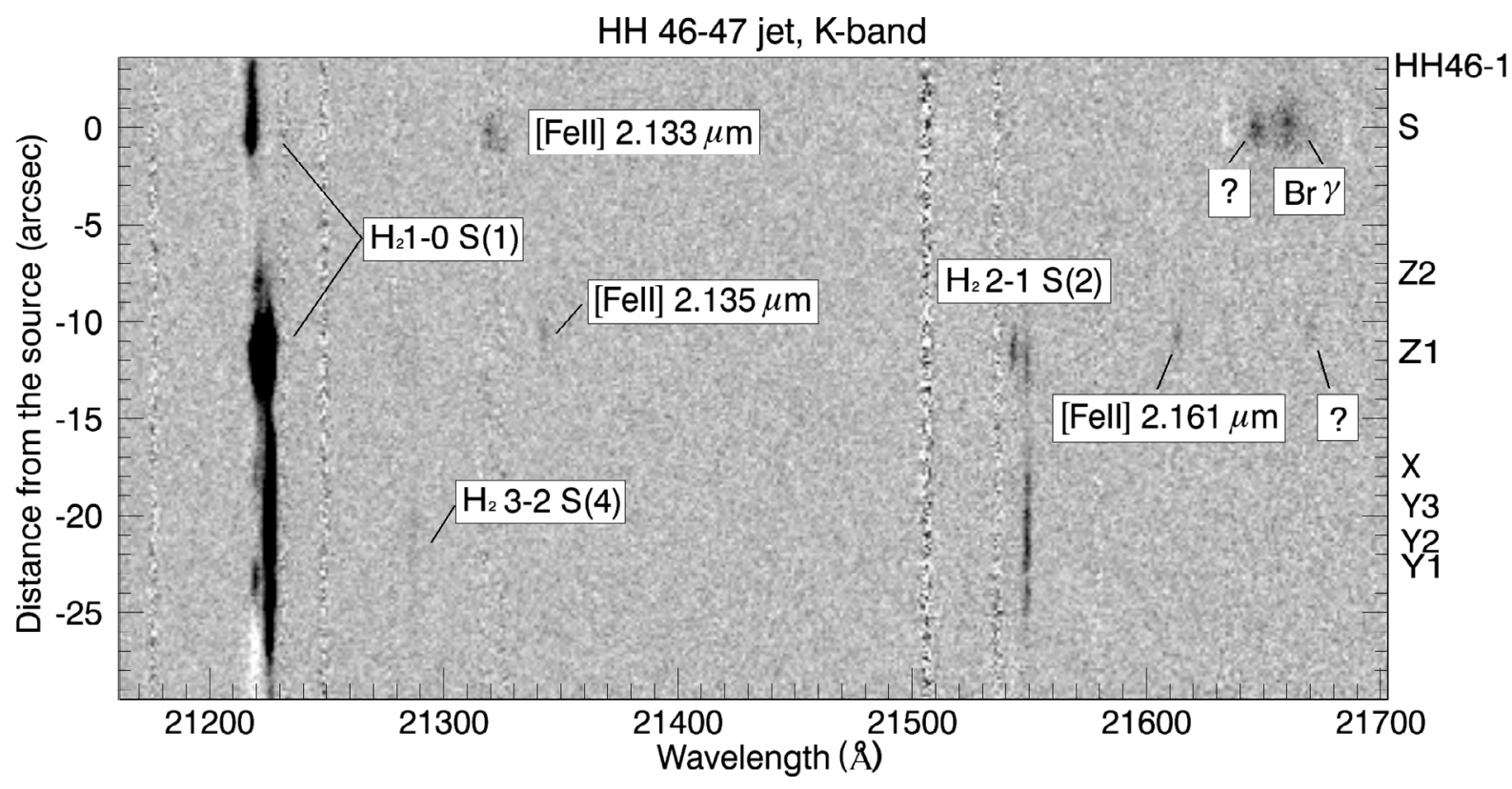

Fig. 3. MR continuum subtracted spectral image of the HH46-47 jet in the $K$-band. The distance from the source is represented on the left-hand $Y$-axis, while the knots are labelled on the right-hand.

Table 3. $A_{V}$ along the HH46-47 jet.

\begin{tabular}{cc}
\hline \hline Knot & $\begin{array}{c}A_{V} \pm \triangle A_{V}{ }^{a} \\
(\mathrm{mag})\end{array}$ \\
\hline ]S & $6.6 \pm 0.2$ \\
Z2 & $12.5 \pm 1.6$ \\
Z1 & $6.1 \pm 0.1$ \\
\hline
\end{tabular}

Notes. (a) Visual extinction measured from the ratio [Fe II] 1.64/1.25 $\mu \mathrm{m}$.

impact of the blue-shifted lobe of the jet with the cavity at $\sim 6^{\prime \prime}$ NE of the source. The same process may occur at the same distance in the red-shifted counter-lobe, giving rise to an increase in extinction.

\section{2. $\mathrm{H}_{2}$ temperature and mass flux}

Using the different $\mathrm{H}_{2}$ detected transitions, excitation diagrams for several knots (Z1, X, Y3, Y2 and Y1) along the jet showing enough $\mathrm{H}_{2}$ lines have been constructed. As an example, Fig. 6 shows the excitation diagram of the knot Z1, the brightest knot observed in HH46-47. The excitation diagrams for the rest of the knots are reported in the Appendix B. In this kind of diagrams the natural logarithm of $N(v, J) / g_{v, J}$ versus the excitation energy $E(v, J)$ of a given level $(v, J)$ is represented, where $N(v, J) / g_{v, J}$ is the ratio of the column density over the statistical weight. The different symbols indicate lines coming from different vibrational levels, while the straight line represents the best linear fit through our data. The slope of the line is proportional to the inverse of the gas temperature. Extinction values as derived from the [Fe II] lines have been adopted. The extinction can, in principle, be also derived from the $\mathrm{H}_{2} 1-0 \mathrm{~S}(1) / 1-0 \mathrm{Q}(3)$ line ratio. But, this method involves lines close in wavelength and thus it is not sensitive enough to extinction variations. In addition, the $\mathrm{Q}$ lines fall in a poor atmospheric transition region (around $\sim 2.3 \mu \mathrm{m}$ ) where their fluxes are affected by significant uncertainties. Still, the fact that our data can be well traced by a straight line on the rovibrational diagram indicates that the extinction values derived from the [Fe II] lines agree well with the $\mathrm{H}_{2}$ ones. The resulting temperature values are indicated in Table 4 and range between $\sim 2000 \mathrm{~K}$ and $\sim 2800 \mathrm{~K}$.

The mass flux carried out by the warm molecular component $\left(\dot{M}_{\mathrm{H}_{2}}\right)$ has been also estimated from the observed $\mathrm{H}_{2}$ transitions in the knots Z1, X, Y3, Y2 and Y1. $\dot{M}_{\mathrm{H}_{2}}$ can be inferred from the relationship $\dot{M}_{\mathrm{H}_{2}}=2 N\left(\mathrm{H}_{2}\right) A \mathrm{~d} v_{t} / \mathrm{d} l_{t}$. Here, $A$ is the area of the emitting region sampled by the slit, $\mathrm{d} l_{t}$ and $\mathrm{d} v_{t}$ are the projected length and the tangential velocity, and $N\left(\mathrm{H}_{2}\right)$ is the total column density. $\dot{M}_{\mathrm{H}_{2}}$ has been derived in the same spatial region extracted for the [Fe II] line analysis. The $\mathrm{d} v_{t}$ value has been taken from Eislöffel \& Mundt (1994), while $N\left(\mathrm{H}_{2}\right)$ for each knot has been found from the intercept to zero of the straight line fitted to the $\mathrm{H}_{2}$ transitions in the Boltzmann diagram. The derived values for each knot are reported in Table 4. The average $\dot{M}_{\mathrm{H}_{2}}$ value along the jet is $\sim 3.8 \times 10^{-9} M_{\odot} \mathrm{yr}^{-1}$. This estimate is however a lower limit, since we are only considering the warm component of the gas.

\subsection{Electron density}

The electron density $\left(n_{\mathrm{e}}\right)$ can be derived from [Fe II] lines coming from levels with similar excitation energies. This is the case of the ratio [Fe II] $1.600 / 1.644 \mu \mathrm{m}$, which is sensitive to $n_{\mathrm{e}}$ values between $10^{3}-10^{5} \mathrm{~cm}^{-3}$ and weakly dependent on the temperature. This ratio has been fitted with a NLTE code including the first 16 fine-structure levels of the [Fe II] (Nisini et al. 2002).

The electron density has been derived by integrating the total flux of the line profile of every extracted knot for both lines. The $n_{\mathrm{e}}$ values and the intervals used to extract the knots are reported in Table 5. Previous studies on protostellar jets like HH1, HH111 and HH34, have shown evidences of a decrease of the electron density with distance (see, e.g., Bacciotti \& Eislöffel 1999; Nisini et al. 2005; Podio et al. 2006). The same behaviour may also occur in HH46-47. Indeed the electron density roughly decreases from a value of $6300 \mathrm{~cm}^{-3}$ in knot $\mathrm{S}$ to a value of 

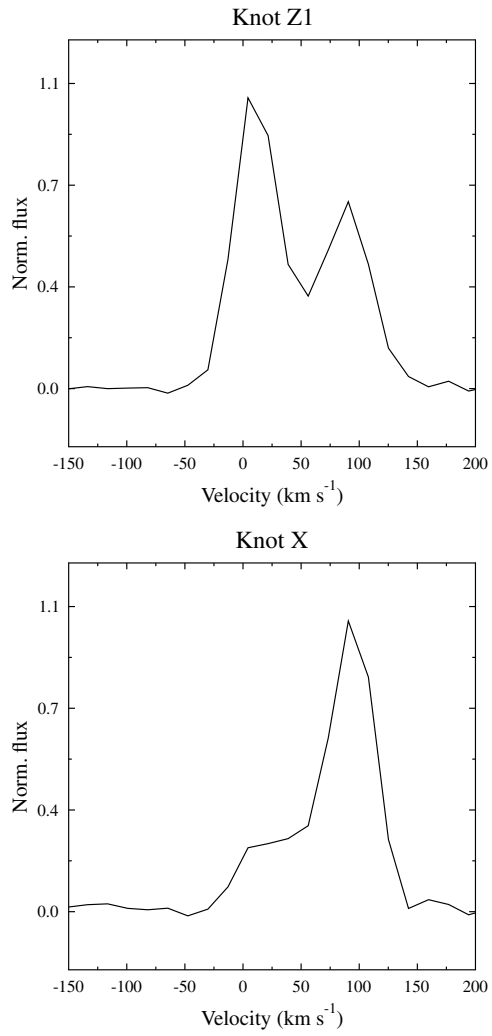

Fig. 4. Normalised line profiles of the $\mathrm{H}_{2} 2.122 \mu \mathrm{m}$ line for knots Z1 (upper panel) and $X$ (lower panel). In the $X$-axis the LSR radial velocity is represented. The velocities are corrected from a cloud velocity of $+20 \mathrm{~km} \mathrm{~s}^{-1}$ (Hartigan et al. 1993)

$3200 \mathrm{~cm}^{-3}$ in knot Z1. These two knots are displaced, however, in different lobes of the jet. In addition, the increase of $n_{\mathrm{e}}$ from knot Z2 to knot Z1 and the the lack of further [Fe II] emitting knots does not allow us to properly probe the dependence on distance of $n_{\mathrm{e}}$.

\section{4. [Fe II] depletion}

Refractory species as iron are expected to be locked into dust grains in the quiescent conditions of molecular clouds. Still, high velocity shocks inside the jet beam are supposed to destroy the grains and release $\mathrm{Fe}$ atoms in gaseous form. In recent years several studies have been done to quantify the presence of dust along protostellar jets and measure the shock efficiency in disrupting the dust grain cores (see e.g., Nisini et al. 2005; Podio et al. 2006; Giannini et al. 2008). The iron gas phase abundance can be measured comparing the emission of [Fe II] with that of a non-refractory species of a known abundance. A very suitable ratio is the one between the [Fe II] $1.257 \mu \mathrm{m}$ line and the [P II] $1.188 \mu \mathrm{m}$ line (Oliva et al. 2001). These two lines are very close in wavelength and thus their ratio is poorly affected by extinction. In addition, both lines are excited under similar conditions and can be assumed in the first ionised state at a similar percentage. Assuming a solar abundance for both elements, the expected theoretical line ratio is $[\mathrm{Fe}$ II $] /[\mathrm{P} \mathrm{III}] \sim 1 / 2[\mathrm{Fe} / \mathrm{P}] \sim 56$ (Oliva et al. 2001). Thus, observed values lower than this indicate that some of the iron is locked in grains.

Along the HH46-47 jet, both the [Fe II] $1.257 \mu \mathrm{m}$ and the [P II] $1.188 \mu \mathrm{m}$ lines have been detected in knots $\mathrm{S}$ and $\mathrm{Z} 1$. The measured $[\mathrm{Fe} \mathrm{II}] /[\mathrm{P} \mathrm{II}]$ ratio is $\sim 7.3$ in knot $\mathrm{S}$ and $\sim 25.6$ in
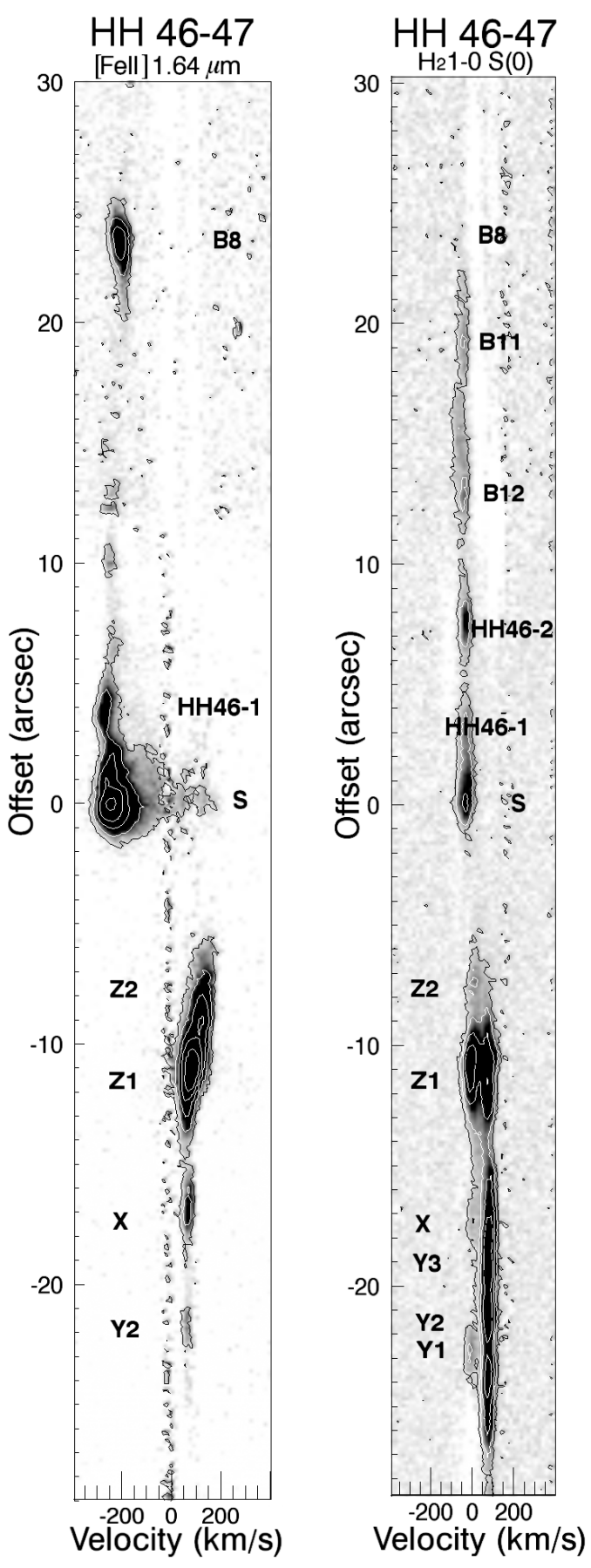

Fig. 5. Continuum-subtracted PVDs for the [Fe II] $1.644 \mu \mathrm{m}$ and $\mathrm{H}_{2} 2.122 \mu \mathrm{m}$ lines along the HH46-47 jet. Contours show values of 3, $9, \ldots, 243 \sigma$ for the $\left[\mathrm{Fe}\right.$ II] line and $4.5,13.5, \ldots, 364.5 \sigma$ for the $\mathrm{H}_{2}$. On the $Y$ axis the distance from the source HH46-IRS is reported in arcsec.

knot Z1. These values indicate that around $88 \%$ and $58 \%$ of the iron is still in dust grains in knots $\mathrm{S}$ and $\mathrm{Z1}$, respectively. This leads to a ratio $(\mathrm{Fe} / \mathrm{H})_{\text {gas }} \sim 3.92 \times 10^{-6}$ in the knot $\mathrm{S}$ and a $(\mathrm{Fe} / \mathrm{H})_{\mathrm{gas}} \sim 1.18 \times 10^{-5}$ in the knot $\mathrm{Z} 1$. The strong iron depletion at the jet base is still lower than the $99 \%$ diffuse gas iron depletion (see, e.g., Savage \& Sembach 1996), indicating that a small fraction of the iron has come back into gas phase at the base of the jet with respect to the interstellar value. Evidence that the gas phase iron abundance is low in knots close to the source and increases with the distance has been also found in the HH1 jet (Nisini et al. 2005). In knot Z1, the [Ti II] $1.140 \mu \mathrm{m}$ line has also 
Table 4. $\mathrm{d} v_{t}, T, N\left(\mathrm{H}_{2}\right), \dot{M}_{\mathrm{H}_{2}}$ along the HH46-47 jet.

\begin{tabular}{ccccc}
\hline \hline Knot & $\begin{array}{c}\mathrm{d} v_{t}{ }^{a} \\
\left(\mathrm{~km} \mathrm{~s}^{-1}\right)\end{array}$ & $\begin{array}{c}T^{b} \\
(\mathrm{~K})\end{array}$ & $\begin{array}{c}N\left(\mathrm{H}_{2}\right)^{c} \\
\left(10^{17} \mathrm{~cm}^{-2}\right)\end{array}$ & $\begin{array}{c}\dot{M}_{\mathrm{H}_{2}}{ }^{d} \\
\left(M_{\odot} \mathrm{yr}^{-1}\right)\end{array}$ \\
\hline $\mathrm{Z} 1$ & 131 & $2797 \pm 100$ & 3.3 & $4.5 \times 10^{-9}$ \\
$\mathrm{X}$ & 19 & $2078 \pm 42$ & 3.7 & $7.3 \times 10^{-10}$ \\
$\mathrm{Y} 3$ & 122 & $2564 \pm 53$ & 3.6 & $4.6 \times 10^{-9}$ \\
$\mathrm{Y} 2$ & 122 & $2330 \pm 50$ & 5.1 & $6.4 \times 10^{-9}$ \\
$\mathrm{Y} 1$ & 122 & $2093 \pm 42$ & 2.4 & $3.0 \times 10^{-9}$ \\
\hline
\end{tabular}

Notes. ${ }^{(a)}$ Tangential velocities from Eislöffel \& Mundt (1994). ${ }^{(b)} \mathrm{H}_{2}$ temperature. ${ }^{(c)} \mathrm{H}_{2}$ column density. ${ }^{(d)} \mathrm{H}_{2}$ mass ejection rate.

been detected. Ti is expected to be fully ionised in the jet plasma, since it has a low ionisation potential of $6.82 \mathrm{eV}$. Like iron, the titanium is a high-refactory element, and thus the Fe/Ti ratio can give us some clues about the relative abundance of both elements as well as about the selective depletion pattern of both elements in the dust along protostellar jets. With this aim we have computed the [Fe II] $1.257 \mu \mathrm{m} /[\mathrm{Ti}$ II $] 1.140 \mu \mathrm{m}$ intensity ratio using an NLTE model for these species (Garcia Lopez et al. 2008). The observed ratio, assuming the physical conditions present in knot $\mathrm{Z1}$ is $\sim 70$, indicates a Fe/Ti ratio five times smaller than the one theorically expected of $[\mathrm{Fe} / \mathrm{Ti}]_{\odot}=354$, using the solar abundances of Asplund et al. (2005). The small Fe/Ti is consistent with what is found in the HH1 jet (Garcia Lopez et al. 2008), where a Fe/Ti ratio two to three times lower than solar was derived.

\section{Medium resolution spectral analysis}

\subsection{Electron density and mass flux}

The [Fe II] 1.600 and $1.644 \mu \mathrm{m}$ lines have also been detected at medium resolution in the knots $\mathrm{S}, \mathrm{Z} 1$ and $\mathrm{Z} 2$. In these knots we can test how the electron density varies as a function of the radial velocity by measuring the $1.600 / 1.644 \mu \mathrm{m}$ ratio in each pixel along the spectral profile. The [Fe II] ratio was computed only for the velocity points where the intensity is greater than $S / N \sim 3$. Figure 7 shows electron density variations along the MR spectral profile of the knots S, Z1 and Z2 (upper panels) together with the normalised line profile of the [Fe II] $1.600 \mu \mathrm{m}$ and $1.644 \mu \mathrm{m}$ lines (lower panels). The electron density increases as the velocity decreases in all of the knots where two velocity components were detected in [Fe II]. In particular, the knot $\mathrm{S}$ close to the source has a behaviour very similar to what was found in HH34 (Garcia Lopez et al. 2008), with the electron densities higher in the gas at low velocity. We have separately measured the $n_{\mathrm{e}}$ in the HVC and LVC components. Near to the source (knot $\mathrm{S}$ ), we have defined a HVC ranging from $\sim-239 \mathrm{~km} \mathrm{~s}^{-1}$ to $\sim-181 \mathrm{~km} \mathrm{~s}^{-1}$ (consistent with the FWHM of a Gaussian-fit through the line), and an LVC from $\sim-181 \mathrm{~km} \mathrm{~s}^{-1}$ to $\sim-50 \mathrm{~km} \mathrm{~s}^{-1}$. Far from the source, only the knot Z2 shows a clear double velocity component: here we have defined the intervals from $\sim 110 \mathrm{~km} \mathrm{~s}^{-1}$ to $\sim 167 \mathrm{~km} \mathrm{~s}^{-1}$ and from $\sim 80 \mathrm{~km} \mathrm{~s}^{-1}$ to $\sim 110 \mathrm{~km} \mathrm{~s}^{-1}$ for the high and low velocity gas, respectively.

The $n_{\mathrm{e}}$ values for the integrated velocity components are reported in Table 5. The electron density of the LVC $\left(n_{\mathrm{e}}(\mathrm{LVC}) \sim 4600-7600\right)$ is higher than the electron density of the HVC $\left(n_{\mathrm{e}}(\mathrm{HVC}) \sim 2300-5000\right)$ as evidenced by Fig. 7 . The behaviour found in knot $\mathrm{S}$ is similar to what we have already found at the HH34 jet base and contrasts with the results for T Tauri

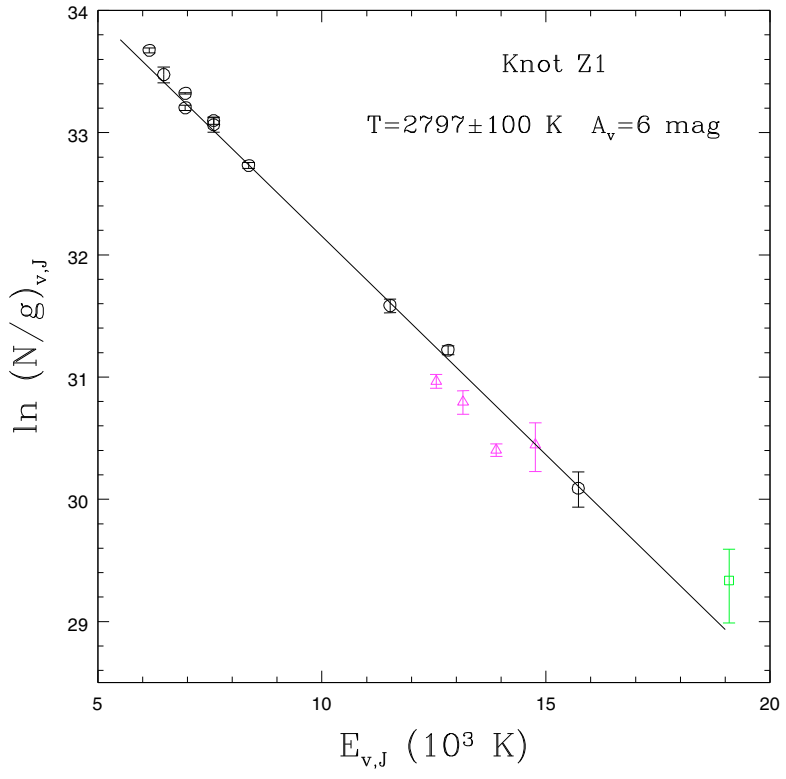

Fig. 6. $\mathrm{H}_{2}$ rotational diagram for the different lines in the knot $\mathrm{Z} 1$ of HH46-47. Different symbols indicate lines coming from different vibrational levels: circles indicate $v=1$, triangles $v=2$ and squares $v=3$. The straight line represents the best fit through the data. The corresponding temperature and the adopted extinction value is indicated.

stars, where the highest electron density is associated with the high velocity gas pertaining to the more collimated inner jet section, while the low velocity component is a less collimated gas at lower density (e.g., Coffey et al. 2008). These contrasting results may reflect different excitation conditions in the structure of Class I jets or may be a result biased by the low spatial resolution of our observations that does not allow us to resolve the same spatial scales as those probed in T Tauri jets, namely within 100-200 AU.

At the bow shock positions far from the source, like in the $\mathrm{Z} 2$ knot, variations of the electron density with velocity may depend on the bow inclination angle and the section of the bow intercepted by the slit, as shown by Indebetouw \& Noriega-Crespo (1995). According to this model, a bow shock seen on-axis at an inclination angle of 30 degree, as in our case, shows qualitatively a behaviour like the one we observe, namely with the highest electron densities displaced at low velocity.

In order to compute the contribution to the mass flux of the atomic component, we have derived $\dot{M}_{\text {jet }}$ from the luminosity of the [Fe II] $1.644 \mu \mathrm{m}$ line, following the approach described in Garcia Lopez et al. (2008). To compute the theoretical [Fe II] lines fractional population, the $n_{\mathrm{e}}$ values derived separately for the LV and HV components have been assumed. A constant value of $10000 \mathrm{~K}$ have instead been assumed for the electron temperature: the derived populations are only a little sensitive to this value and the results do not change significantly if the temperature is maintained between $\sim 7000 \mathrm{~K}$ and $15000 \mathrm{~K}$. An inclination angle of the jet with respect to the plane of the sky $i \sim 34^{\circ}$ (Eislöffel \& Mundt 1994) has been assumed and used to infer the velocity of the knots projected perpendicular to the line of sight through our measurements of the radial velocity. These values are reported in Table 6, while the adopted length of the knots projected perpendicular to the line of sight is listed in Table 5. The [Fe II] $1.644 \mu \mathrm{m}$ line flux has been de-reddened using the extinction derived from the ratio [Fe II] 1.644/1.257 and reported in Table 3. 
Table 5. Electron density along the HH46-47 jet.

\begin{tabular}{ccccc}
\hline \hline Knot & $\begin{array}{c}r_{t}{ }^{a} \\
\left({ }^{\prime \prime}\right)\end{array}$ & $\begin{array}{c}n_{\mathrm{e}} \pm \Delta n_{\mathrm{e}}{ }^{b} \\
\left(\mathrm{~cm}^{-3}\right)\end{array}$ & $\begin{array}{c}n_{\mathrm{e}}(\mathrm{HVC})^{c} \\
\left(\mathrm{~cm}^{-3}\right)\end{array}$ & $\begin{array}{c}n_{\mathrm{e}}(\mathrm{LVC})^{b} \\
\left(\mathrm{~cm}^{-3}\right)\end{array}$ \\
\hline $\mathrm{S}$ & $(-3.4,+2.8)$ & $6300_{-1300}^{+1700}$ & 5400 & 7650 \\
$\mathrm{Z} 2$ & $(-9.3,-5)$ & $1300_{-1200}^{+1800}$ & 2350 & 4600 \\
$\mathrm{Z} 1$ & $(-14.8,-9.3)$ & $3200_{-850}^{+700}$ & 3100 & \\
\hline
\end{tabular}

Notes. ${ }^{(a)}$ Distance from the source in arcsec. Negative values correspond to the southeastern, redshifted jet axis. ${ }^{(b)}$ Electron density measured from the ratio [Fe II] 1.60/1.64 $\mu \mathrm{m} .{ }^{(c)}$ Electron density measured from the ratio [Fe II] $1.60 / 1.64 \mu \mathrm{m}$ for the HVC and LVC.

Table 6. $\dot{M}_{\text {jet }}$ along the HH46-47 jet.

\begin{tabular}{ccccc}
\hline \hline Knot & $\begin{array}{c}\mathrm{d} v_{t}(\mathrm{HVC})^{a} \\
\mathrm{~km} \mathrm{~s}^{-1}\end{array}$ & $\begin{array}{c}\dot{M}_{\mathrm{jet}}(\mathrm{HVC})^{b} \\
10^{-7} M_{\odot} \mathrm{yr}^{-1}\end{array}$ & $\begin{array}{c}\mathrm{d} v_{t}(\mathrm{LVC})^{a} \\
\mathrm{~km} \mathrm{~s}^{-1}\end{array}$ & $\begin{array}{c}\dot{M}_{\mathrm{jet}}(\mathrm{LVC})^{b} \\
10^{-8} M_{\odot} \mathrm{yr}^{-1}\end{array}$ \\
\hline $\mathrm{S}$ & 316 & $0.3-1.9$ & 205 & $0.5-3.6$ \\
$\mathrm{Z} 2$ & 213 & $0.4-0.9$ & 136 & $0.2-0.4$ \\
$\mathrm{Z} 1$ & 166 & $0.4-1.0$ & & \\
\hline
\end{tabular}

Notes. ${ }^{(a)}$ Tangential velocity derived from the observed radial velocities assuming an orientation angle of the jet with respect to the plane of the sky of $34^{\circ}$ (Eislöffel \& Mundt 1994). ${ }^{(b)} \dot{M}_{\text {jet }}$ measured from the luminosity of the [Fe II] $1.64 \mu \mathrm{m}$ for the HVC and LVC. The lower value has been derived assuming an iron solar abundance, while the upper value has been inferred assuming the iron gas-phase abundance derived from the $[\mathrm{Fe} \mathrm{II}] /[\mathrm{P}$ II $]$ ratio.

$\dot{M}_{\text {jet }}$ values have been derived both assuming a solar abundance of iron and considering the gas-phase Fe abundance measured from the $\mathrm{Fe} / \mathrm{P}$ analysis, in the assumption that the same dust depletion factor is valid for both the HVC and LVC. The corresponding range of values are listed in Table 6. Values of the order of $0.5-1 \times 10^{-7} M_{\odot} \mathrm{yr}^{-1}$ are found in the HVC, while the LVC shows $\sim 16$ times lower values.

\section{Discussion}

The $[\mathrm{Fe} \mathrm{II}]$ and $\mathrm{H}_{2}$ emission structure at the base of HH46-47 is very similar to the one observed in several Class I jets (e.g., Davis et al. 2001b, 2003; Takami et al. 2006). As in the case of CTTS jets, the forbidden-emission line (FEL) regions of Class I jets are characterised by the presence of two velocity components at the jet base at high and low velocity. The MHEL regions as traced by $\mathrm{H}_{2}$ emission show on contrast just a LVC near the source while higher velocity $\mathrm{H}_{2}$ is usually detected further downstream. In particular the kinematics and physical parameters of the HH46-47 jet are very similar to those found in our previous work on HH34 (Garcia Lopez et al. 2008), where the physical properties have also been derived as a function of velocity. For instance, Fig. 8 shows the [Fe II] 1.644 $\mu \mathrm{m}$ PVDs of the inner region of these two jets. Since the two objects are at a similar distance (i.e. $450 \mathrm{pc}$ ), the emission regions have the same physical extent in this figure. In both the PVDs, the [Fe II] lines broaden and emission at lower velocities down to $0 \mathrm{~km} \mathrm{~s}^{-1}$ appears within $\sim 2^{\prime \prime}$ from the central source, i.e. up to distances of $\sim 1000 \mathrm{AU}$. The extent of the LVC is comparable with the typical size of the envelope of Class I sources (see, e.g., Andre \& Montmerle 1994). Thus this result may indicate an interaction of a low-velocity disc-wind with the source envelope as the possible origin of the LVC in Class I jets. The large extent of the LVC
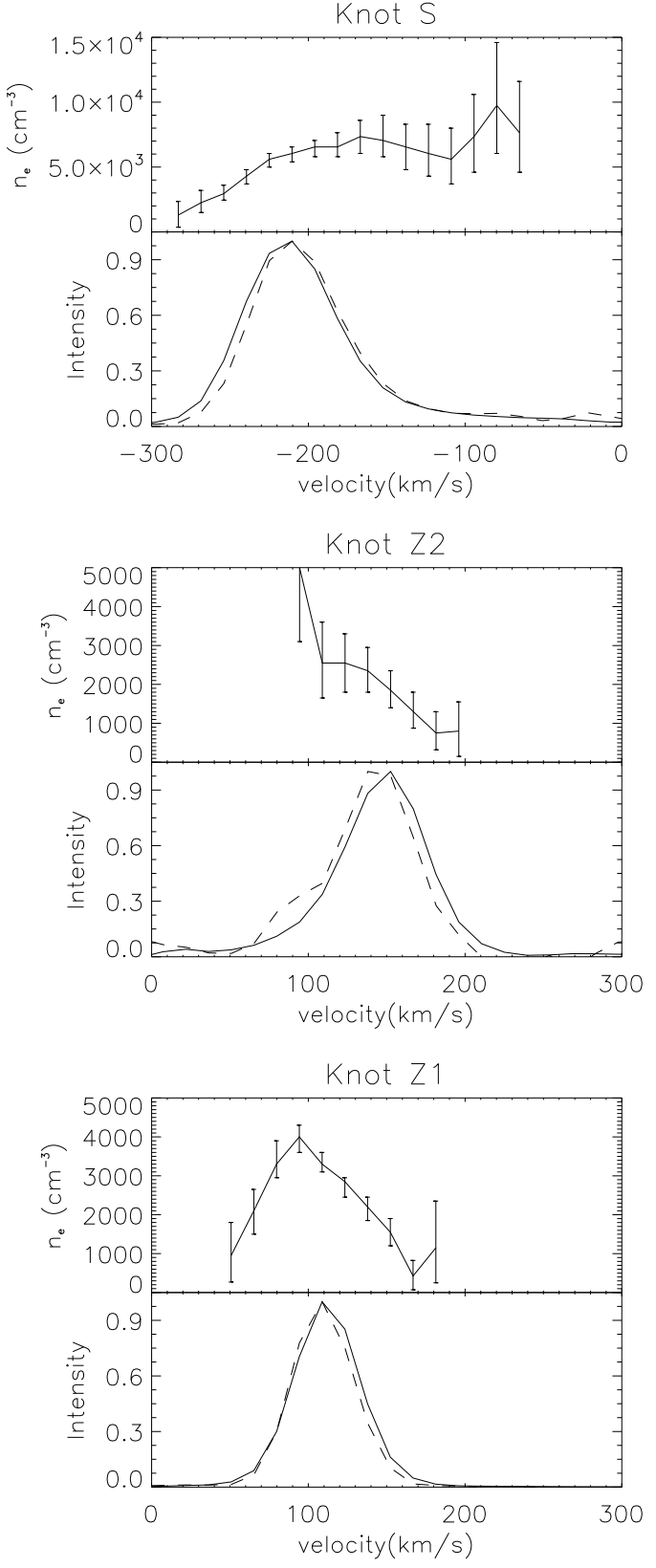

Fig. 7. Normalised to one line profiles (lower panels) of the [Fe II] lines $1.644 \mu \mathrm{m}$ (solid line) and $1.600 \mu \mathrm{m}$ (dotted line), and the electron density in each velocity channel (upper panels) for different extracted knots along the HH46-47 jet.

is at variance with the PVDs of most of the CTT, where the LVC is usually confined within $\sim 200 \mathrm{AU}$ from the source (Pyo et al. 2003, 2006).

The values of electron density and mass flux rate that we derive in HH46-47 and their dependence on the velocity are also similar to those inferred on $\mathrm{HH} 34$, i.e., $n_{\mathrm{e}} \sim 0.5-1 \times 10^{4} \mathrm{~cm}^{-3}$ in the HVC and $\sim 1-2 \times 10^{4} \mathrm{~cm}^{-3}$ in the LVC, and $\dot{M}_{\text {jet }}$ of the order $10^{-7} M_{\odot} \mathrm{yr}^{-1}$. Similar values have also been estimated on a number of Class I YSO jets (e.g., Davis et al. 2003; Podio et al. 2006; Takami et al. 2006).

At variance with $[\mathrm{Fe} \mathrm{II}]$, the $\mathrm{H}_{2}$ PVDs of HH46-47 shows a different behaviour with respect to the one of HH34. In HH34 the $\mathrm{H}_{2}$ PVD shows spatially- and kinematically-separated LVC and HVC, and only the LVC is visible down to the central source. In HH46-47, on the contrary, a single velocity component near the 

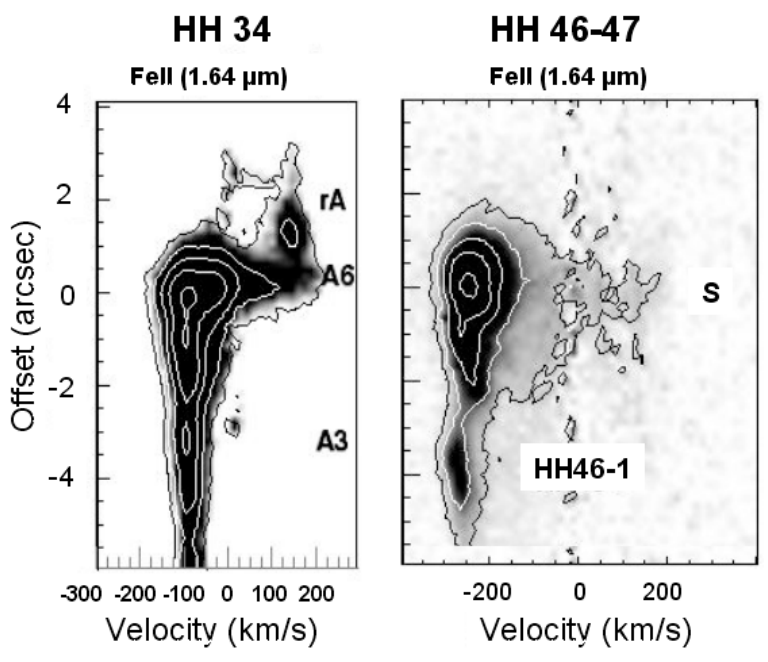

Fig. 8. PVD of the [Fe II] $1.644 \mu \mathrm{m}$ line for the HH34 and HH46-47 jets.

source is detected. The morphology and kinematics of the $\mathrm{H}_{2}$ close to the source (the MHEL regions; Davis et al. 2001b) in Class I objects varies indeed significantly from source to source (Davis et al. 2001b, 2002) and this is another element that makes the origin of the MHEL regions unclear.

In Garcia Lopez et al. (2008) we have compared the [Fe II] $1.644 \mu \mathrm{m}$ PVDs of HH34 with those of the synthetic PVDs available for the disc-wind and $\mathrm{X}$-wind models (Pesenti et al. 2004; Shang et al. 1998). Most of this discussion is also valid for the HH46-47 jet. Briefly, none of these models are able to explain the persistency of the LVC at large distances from the launching regions. This may suggest that the low velocity that we observe far from the source represents dense gas entrained by the high-velocity collimated jet rather than directly ejected gas from the acceleration region.

From the observed HH46-47 peak radial velocity of the $\mathrm{HVC}$, corrected for the inclination angle, we estimate a jet poloidal velocity around $350 \mathrm{~km} \mathrm{~s}^{-1}$. This value is just at the border of the range of poloidal velocities predicted by $\mathrm{X}$-wind models and it also agrees with disc-wind models with launching radii in the range of 0.07-0.15 AU and magnetic lever arms larger than $\sim 5$ (Ferreira et al. 2006).

Further constraints on MHD launching models may be derived from the presence of dust in the initial beam of the HH46-47 jet, as suggested by Podio et al. (2009). We have in fact seen that around $88 \%$ of the iron within $450 \mathrm{AU}$ from the source is depleted, implying that a large fraction of dust grains are still present at the jet base. Dust grains of the ambient medium are expected to be destroyed by the passage of the initial leading bowshock that has velocities higher than $100 \mathrm{~km} \mathrm{~s}^{-1}$ (Draine 2003). Since the amount of iron depletion decreases toward the external knots (only $58 \%$ of iron is found to be depleted in grains at $\sim 5000$ AU from the source), dust grains are unlikely to come from entrained material. In addition they cannot be reformed along the jet beam since the time required for grain reformation is $\sim 10^{7}-10^{8} \mathrm{yr}$ (Jones 2001), while the jet takes $\sim 1300 \mathrm{yr}$ to travel from the central source to the final bow-shock (Hartigan et al. 2005). Therefore, the larger amount of grains near the source may suggest that they have been removed from the accretion disc and transported through the jet. Interestingly it has been shown that in the case of Herbig stars and T Tauri stars the disc is populated by dust grains only beyond the so-called dust evaporation point, $R_{\text {evp }}$ (Akeson et al. 2005; Eisner et al. 2007b,a).
Future similar measurements of $R_{\text {evp }}$ for Class 0/I sources would help to constrain the region from which dusty jets are launched.

\section{Conclusions}

We have presented medium resolution (MR) (in the $H$ - and $K$-band) and low resolution (LR) (in the $J$-, $H$ - and $K$-band) spectra of the Class I driven HH46-47 jet. These observations allow us to resolve two velocity components at the jet base and to study the kinematics and physical properties of the jet as a function of velocity. From the [Fe II] $1.644 / 1.600 \mu \mathrm{m}$ lines the electron density has been derived along the jet and as a function of velocity. In addition, from the luminosity of the [Fe II] $1.644 \mu \mathrm{m}$ line the mass flux for the different velocity components has been derived. Finally, the $[\mathrm{Fe}$ II $] /[\mathrm{P} \mathrm{II}]$ ratio has been used to retrieve important information about the dust depletion inside the jet beam. We summarise our results as follows:

- We were able to trace the HH46-47 jet down to the central source. While the atomic component shows a HVC and LVC near the source, only a single velocity component has been detected for the molecular gas. Within $1^{\prime \prime}$ from the source, the atomic emission broadens down to $0 \mathrm{~km} \mathrm{~s}^{-1}$ and even reaches red-shifted velocities.

- From the ratio of the [Fe II] 1.644, $1.257 \mu$ m lines detected in the LR spectra, the visual extinction for different knots have been derived. We have found an $A_{V}$ ranging from $\sim 6$ mag to $\sim 12$ mag along the jet.

- Several $\mathrm{H}_{2}$ lines have been detected from the LR spectra, allowing us to construct excitation diagrams for different knots. From these diagrams we have derived the temperature and mass flux for the warm molecular component of the jet. The temperature has an average value around $\sim 2300 \mathrm{~K}$, while we have found an average mass flux for the warm molecular component of $\gtrsim 3.7 \times 10^{-9} M_{\odot} \mathrm{yr}^{-1}$.

- The electron density, derived from the [Fe II] 1.600/1.644 $\mu \mathrm{m}$ ratio, roughly decreases from the internal to the more external knots from 9400 to $3200 \mathrm{~cm}^{-3}$. Larger electron density values are found in the LVC with respect to the HVC for all the extracted knots, with an average value of $\sim 6000$ and $\sim 4000 \mathrm{~cm}^{-3}$ for the LVC and HVC, respectively.

- From the $[\mathrm{Fe} \mathrm{II}] /[\mathrm{P}$ II $]$ ratio, we have estimated the iron depletion in the knots S and Z1. We have found that around $88 \%$ and $58 \%$ of the iron is depleted in grains in the knot S and Z1.

- The mass flux rate transported by the [Fe II] has been derived for both the velocity components assuming the $\mathrm{Fe}$ gas-phase abundance derived in this paper. We found that the HVC is transporting the bulk of the mass along the jet with average values of $\dot{M}_{\text {jet }}(\mathrm{HVC}) \sim 1.2 \times 10^{-7} M_{\odot} \mathrm{yr}^{-1}$ and $\dot{M}_{\text {jet }}(\mathrm{LVC}) \sim 2 \times 10^{-8} M_{\odot} \mathrm{yr}^{-1}$. As in the case of HH34 (Garcia Lopez et al. 2008), the difference in the tangential velocity between the HVC and LVC is not enough to account for the much hihger $\dot{M}_{\text {jet }}$ value in the HVC, indicating a significantly larger value of the $\left(n_{\mathrm{H}} V\right)^{4}$ product in the HVC than in the LVC (from a factor $\sim 1.5$ in the knot $\mathrm{Z} 2$ to $\sim 3.5$ in knot S).

- Many of the derived properties of the HH46-47 are common to jets from YSOs in different evolutionary states. The derived densities and mass flux values are typical of Class I objects or very active T Tauri stars. However, the observed spatial extent of the LVC and the velocity dependence of the

\footnotetext{
${ }^{4}$ Where $n_{\mathrm{H}}$ and $V$ are the total density and the emitting volume, see Garcia Lopez et al. (2008) for more details.
} 
electron density are not explained by the current jet formation models.

Acknowledgements. The present work was partly supported by the European Communitys Marie Curie Actions - Human Resource and Mobility within the JETSET (Jet Simulations, Experiments and Theory) network under contract MRTN-CT-2004 005592. L.P. work was funded by a Fellowship from the Irish Research Council for Science, Engineering and Technology.

\section{References}

Akeson, R. L., Boden, A. F., Monnier, J. D., et al. 2005, ApJ, 635, 1173 Andre, P., \& Montmerle, T. 1994, ApJ, 420, 837 Antoniucci, S., Nisini, B., Giannini, T., \& Lorenzetti, D. 2008, A\&A, 479, 503 Asplund, M., Grevesse, N., \& Sauval, A. J. 2005, in Cosmic Abundances as Records of Stellar Evolution and Nucleosynthesis, ed. T. G. Barnes, III, \& F. N. Bash, ASP Conf. Ser., 336, 25

Bacciotti, F., \& Eislöffel, J. 1999, A\&A, 342, 717

Bacciotti, F., Mundt, R., Ray, T. P., et al. 2000, ApJ, 537, L49

Coffey, D., Bacciotti, F., \& Podio, L. 2008, ApJ, 689, 1112

Davis, C. J., Hodapp, K. W., \& Desroches, L. 2001a, A\&A, 377, 285

Davis, C. J., Ray, T. P., Desroches, L., \& Aspin, C. 2001b, MNRAS, 326, 524

Davis, C. J., Stern, L., Ray, T. P., \& Chrysostomou, A. 2002, A\&A, 382, 1021

Davis, C. J., Whelan, E., Ray, T. P., \& Chrysostomou, A. 2003, A\&A, 397, 693

Davis, C. J., Nisini, B., Takami, M., et al. 2006, ApJ, 639, 969

Draine, B. T. 2003, ARA\&A, 41, 241

Eislöffel, J., \& Mundt, R. 1994, A\&A, 284, 530

Eislöffel, J., Davis, C. J., Ray, T. P., \& Mundt, R. 1994, ApJ, 422, L91

Eisner, J. A., Chiang, E. I., Lane, B. F., \& Akeson, R. L. 2007a, ApJ, 657, 347

Eisner, J. A., Hillenbrand, L. A., White, R. J., et al. 2007b, ApJ, 669, 1072

Fernandes, A. J. L. 2000, MNRAS, 315, 657

Ferreira, J. 1997, A\&A, 319, 340
Ferreira, J., Dougados, C., \& Cabrit, S. 2006, A\&A, 453, 785

Garcia Lopez, R., Nisini, B., Giannini, T., et al. 2008, A\&A, 487, 1019

Giannini, T., Calzoletti, L., Nisini, B., et al. 2008, A\&A, 481, 123

Hartigan, P., Morse, J. A., Heathcote, S., \& Cecil, G. 1993, ApJ, 414, L121

Hartigan, P., Heathcote, S., Morse, J. A., Reipurth, B., \& Bally, J. 2005, AJ, 130, 2197

Heathcote, S., Morse, J. A., Hartigan, P., et al. 1996, AJ, 112, 1141

Indebetouw, R., \& Noriega-Crespo, A. 1995, AJ, 109, 752

Jones, A. P. 2001, Roy. Soc. London Philos. Trans. Ser. A, 359, 1961

Lavalley, C., Cabrit, S., Dougados, C., Ferruit, P., \& Bacon, R. 1997, A\&A, 327, 671

Nishikawa, T., Takami, M., Hayashi, M., Wiseman, J., \& Pyo, T.-S. 2008, ApJ, 684,1260

Nisini, B., Caratti o Garatti, A., Giannini, T., \& Lorenzetti, D. 2002, A\&A, 393, 1035

Nisini, B., Bacciotti, F., Giannini, T., et al. 2005, A\&A, 441, 159 (N05)

Nussbaumer, H., \& Storey, P. J. 1988, A\&A, 193, 327

Oliva, E., Marconi, A., Maiolino, R., et al. 2001, A\&A, 369, L5

Pesenti, N., Dougados, C., Cabrit, S., O'Brien, D., \& Garcia, P. J. V. 2004, Ap\&SS, 292, 539

Podio, L., Bacciotti, F., Nisini, B., et al. 2006, A\&A, 456, 189 (P06)

Podio, L., Medves, S., Bacciotti, F., Eislöffel, J., \& Ray, T. P. 2009, A\&A, 506, 779

Pyo, T.-S., Kobayashi, N., Hayashi, M., et al. 2003, ApJ, 590, 340

Pyo, T.-S., Hayashi, M., Kobayashi, N., et al. 2006, ApJ, 649, 836

Quinet, P., Le Dourneuf, M., \& Zeippen, C. J. 1996, A\&AS, 120, 361

Reipurth, B., Yu, K. C., Heathcote, S., Bally, J., \& Rodríguez, L. F. 2000, AJ, 120, 1449

Savage, B. D., \& Sembach, K. R. 1996, ApJ, 470, 893

Schultz, A. S. B., Burton, M. G., \& Brand, P. W. J. L. 2005, MNRAS, 358, 1195

Shang, H., Shu, F. H., \& Glassgold, A. E. 1998, ApJ, 493, L91

Shu, F. H., Najita, J., Ostriker, E. C., \& Shang, H. 1995, ApJ, 455, L155

Takami, M., Chrysostomou, A., Ray, T. P., et al. 2006, ApJ, 641, 357 
R. Garcia Lopez et al.: Kinematics and physical characteristics of HH46-47

\section{Appendix A: Knot fluxes at low resolution}

Table A.1. Observed unreddened lines along HH46-47: atomic lines.

\begin{tabular}{|c|c|c|c|c|c|c|}
\hline \multirow[t]{2}{*}{ Line id. } & \multirow[t]{2}{*}{$\lambda(\mu \mathrm{m})$} & \multicolumn{5}{|c|}{$F(\Delta F)\left(10^{-16} \mathrm{erg} \mathrm{s}^{-1} \mathrm{~cm}^{-2}\right)$} \\
\hline & & $\mathrm{S}$ & HH46-1 & B8 & $\mathrm{Z2}$ & $X$ \\
\hline$[\mathrm{P}$ II $]{ }^{3} \mathrm{P}_{2}-{ }^{1} \mathrm{D}_{2}$ & 1.188 & $6.2(1.2)$ & $\cdots$ & $\ldots$ & $\ldots$ & $\ldots$ \\
\hline$[\mathrm{Fe}$ II $] \mathrm{a}^{4} \mathrm{D}_{5 / 2}-\mathrm{a}^{6} \mathrm{D}_{7 / 2}$ & 1.249 & $1.9(0.6)$ & & & $\ldots$ & \\
\hline$[\mathrm{Fe}$ II $] \mathrm{a}^{4} \mathrm{D}_{7 / 2}-\mathrm{a}^{6} \mathrm{D}_{9 / 2}$ & 1.257 & $54.2(0.8)$ & $15.8(0.9)$ & $11.9(0.7)$ & $7.1(0.9)$ & $6.4(1.0)$ \\
\hline$[\mathrm{Fe}$ II $] \mathrm{a}^{2} \mathrm{G}_{9 / 2}-\mathrm{a}^{4} \mathrm{D}_{7 / 2}$ & 1.267 & $3.2(0.8)$ & $\ldots$ & $\ldots$ & $\ldots$ & $\ldots$ \\
\hline$[\mathrm{Fe}$ II $] \mathrm{a}^{4} \mathrm{D}_{1 / 2}-\mathrm{a}^{6} \mathrm{D}_{1 / 2}$ & 1.271 & $2.0(0.3)$ & $\ldots$ & $\ldots$ & $\ldots$ & $\ldots$ \\
\hline$[\mathrm{Fe}$ II $] \mathrm{a}^{4} \mathrm{D}_{3 / 2}-\mathrm{a}^{6} \mathrm{D}_{3 / 2}$ & 1.279 & $2.4(0.6)$ & $\ldots$ & $\ldots$ & $\ldots$ & $\ldots$ \\
\hline$[\mathrm{Fe}$ II $] \mathrm{a}^{4} \mathrm{D}_{5 / 2}-\mathrm{a}^{6} \mathrm{D}_{5 / 2}$ & 1.295 & $8.9(1.1)$ & $\ldots$ & $\ldots$ & $\ldots$ & $\ldots$ \\
\hline$[\mathrm{Fe}$ II $] \mathrm{a}^{4} \mathrm{D}_{3 / 2}-\mathrm{a}^{6} \mathrm{D}_{1 / 2}$ & 1.298 & $3.4(0.9)$ & $\ldots$ & $\ldots$ & $\ldots$ & $\ldots$ \\
\hline$[\mathrm{Fe}$ II $] \mathrm{a}^{2} \mathrm{G}_{7 / 2}-\mathrm{a}^{4} \mathrm{D}_{3 / 2}$ & 1.300 & $1.7(0.6)$ & $\ldots$ & $\ldots$ & $\ldots$ & $\ldots$ \\
\hline$[\mathrm{Fe}$ II $] \mathrm{a}^{4} \mathrm{D}_{7 / 2}-\mathrm{a}^{6} \mathrm{D}_{7 / 2}$ & 1.321 & $13.1(0.4)$ & $4.5(1.4)$ & $3.5(0.5)$ & $3.0(1.2)$ & $2.4(1.2)$ \\
\hline$[\mathrm{Fe}$ II $] \mathrm{a}^{4} \mathrm{D}_{5 / 2}-\mathrm{a}^{6} \mathrm{D}_{3 / 2}$ & 1.328 & $4.3(0.6)$ & $\ldots$ & $\ldots$ & $\ldots$ & $\ldots$ \\
\hline$[\mathrm{Fe}$ II $] \mathrm{a}^{4} \mathrm{D}_{5 / 2}-\mathrm{a}^{4} \mathrm{~F}_{9 / 2}$ & 1.534 & $9.9(1.0)$ & $\ldots$ & $\ldots$ & $0.7(0.4)$ & $\ldots$ \\
\hline$[\mathrm{Fe}$ II $] \mathrm{a}^{4} \mathrm{D}_{3 / 2}-\mathrm{a}^{4} \mathrm{~F}_{7 / 2}$ & 1.600 & $11.2(1.0)$ & $\ldots$ & $\ldots$ & $0.9(0.4)$ & $\ldots$ \\
\hline$[\mathrm{Fe}$ II $] \mathrm{a}^{4} \mathrm{D}_{7 / 2}-\mathrm{a}^{4} \mathrm{~F}_{9 / 2}$ & 1.644 & $85.8(0.7)$ & $8.9(0.5)$ & $8.7(0.8)$ & $20.1(0.5)$ & $4.4(0.5)$ \\
\hline$[\mathrm{Fe}$ II $] \mathrm{a}^{4} \mathrm{D}_{1 / 2}-\mathrm{a}^{4} \mathrm{~F}_{5 / 2}$ & 1.664 & $5.7(0.8)$ & $\ldots$ & $\ldots$ & $1.1(0.7)$ & $\ldots$ \\
\hline$[\mathrm{Fe}$ III $] \mathrm{a}^{4} \mathrm{D}_{5 / 2}-\mathrm{a}^{4} \mathrm{~F}_{7 / 2}$ & 1.677 & $13.3(0.9)$ & $\ldots$ & $\ldots$ & $1.8(0.4)$ & $\ldots$ \\
\hline$[\mathrm{Fe}$ II $] \mathrm{a}^{4} \mathrm{D}_{3 / 2}-\mathrm{a}^{4} \mathrm{~F}_{3 / 2}$ & 1.797 & $5.2(0.9)$ & $\ldots$ & $\ldots$ & $\ldots$ & $\ldots$ \\
\hline$[\mathrm{Fe}$ II $] \mathrm{a}^{4} \mathrm{D}_{5 / 2}-\mathrm{a}^{4} \mathrm{~F}_{5 / 2}$ & 1.800 & $14.3(0.7)$ & $\ldots$ & $\ldots$ & $\ldots$ & $\ldots$ \\
\hline$[\mathrm{Fe}$ II $] \mathrm{a}^{4} \mathrm{D}_{7 / 2}-\mathrm{a}^{4} \mathrm{~F}_{7 / 2}$ & 1.810 & $52.6(1.1)$ & $\ldots$ & $\ldots$ & $\ldots$ & $\ldots$ \\
\hline$[\mathrm{Fe}$ II $] \mathrm{a}^{2} \mathrm{P}_{3 / 2}-\mathrm{a}^{4} \mathrm{P}_{3 / 2}$ & 2.133 & $3.2(0.5)$ & $\ldots$ & $\ldots$ & $\ldots$ & $\ldots$ \\
\hline
\end{tabular}

Table A.2. Observed unreddened lines along HH46-47: $\mathrm{H}_{2}$ lines.

\begin{tabular}{cccccccc}
\hline \hline Line id. & $\lambda(\mu \mathrm{m})$ & \multicolumn{5}{c}{$F(\Delta F)\left(10^{-16} \mathrm{erg} \mathrm{s}^{-1} \mathrm{~cm}^{-2}\right)$} \\
& & $\mathrm{S}$ & $\mathrm{Z} 2$ & $X$ & $\mathrm{Y} 3$ & $\mathrm{Y} 2$ & $\mathrm{Y} 1$ \\
\hline $\mathrm{H}_{2} 2-1 \mathrm{~S}(5)$ & 1.945 & $\ldots$ & $\ldots$ & $\ldots$ & $\ldots$ & $\ldots$ & $2.7(0.7)$ \\
$\mathrm{H}_{2} 1-0 \mathrm{~S}(2)$ & 2.034 & $\ldots$ & $2.1(0.6)$ & $26.6(0.6)$ & $10.0(0.6)$ & $13.2(0.4)$ & $8.7(0.4)$ \\
$\mathrm{H}_{2} 2-1 \mathrm{~S}(3)$ & 2.074 & $\ldots$ & $\ldots$ & $3.7(0.5)$ & $2.3(0.2)$ & $3.9(0.4)$ & $\ldots$ \\
$\mathrm{H}_{2} 1-0 \mathrm{~S}(1)$ & 2.122 & $12.0(0.5)$ & $5.6(0.3)$ & $36.3(0.3)$ & $27.0(0.3)$ & $3.3(0.2)$ & $22.7(0.2)$ \\
$\mathrm{H}_{2} 2-1 \mathrm{~S}(2)$ & 2.154 & $\ldots$ & $\ldots$ & $1.1(0.2)$ & $\ldots$ & $1.6(0.2)$ & $\ldots$ \\
$\mathrm{H}_{2} 1-0 \mathrm{~S}(0)^{a}$ & 2.224 & $12.7(0.6)$ & $3.1(0.6)$ & $7.7(0.4)$ & $5.4(0.5)$ & $6.9(0.3)$ & $5.2(0.2)$ \\
$\mathrm{H}_{2} 2-1 \mathrm{~S}(1)$ & 2.248 & $\ldots$ & $1.4(0.4)$ & $3.4(0.3)$ & $3.0(0.5)$ & $4.6(0.2)$ & $3.5(0.3)$ \\
$\mathrm{H}_{2} 1-0 \mathrm{Q}(1)$ & 2.407 & $16.6(1.3)$ & $4.8(0.6)$ & $28.9(0.6)$ & $20.8(0.8)$ & $24.3(0.7)$ & $17.8(0.5)$ \\
$\mathrm{H}_{2} 1-0 \mathrm{Q}(2)$ & 2.413 & $13.8(1.5)$ & $2.3(0.3)$ & $\ldots$ & $\ldots$ & $\ldots$ & $\ldots$ \\
$\mathrm{H}_{2} 1-0 \mathrm{Q}(3)$ & 2.424 & $16.2(1.3)$ & $5.8(0.5)$ & $30.7(0.6)$ & $23.3(1.5)$ & $26.5(0.7)$ & $17.8(0.5)$ \\
$\mathrm{H}_{2} 1-0 \mathrm{Q}(4)$ & 2.437 & $9.3(1.1)$ & $\ldots$ & $8.4(0.7)$ & $6.1(1.2)$ & $8.0(0.6)$ & $5.2(0.6)$ \\
$\mathrm{H}_{2} 1-0 \mathrm{Q}(5)$ & 2.455 & $\ldots$ & $\ldots$ & $23.4(0.9)$ & $15.7(1.6)$ & $21.6(0.8)$ & $14.9(0.7)$ \\
$\mathrm{H}_{2} 1-0 \mathrm{Q}(6)$ & 2.476 & $\ldots$ & $\ldots$ & & $\ldots$ & $6.6(0.6)$ & $\ldots$ \\
\hline
\end{tabular}

Notes. ${ }^{(a)}$ Blend with $\left[\mathrm{Fe}\right.$ II] $\mathrm{b}^{2} \mathrm{~F}_{5 / 2}-\mathrm{a}^{2} \mathrm{~F}_{7 / 2}$. 


\section{Appendix B: Rovibrational diagrams}
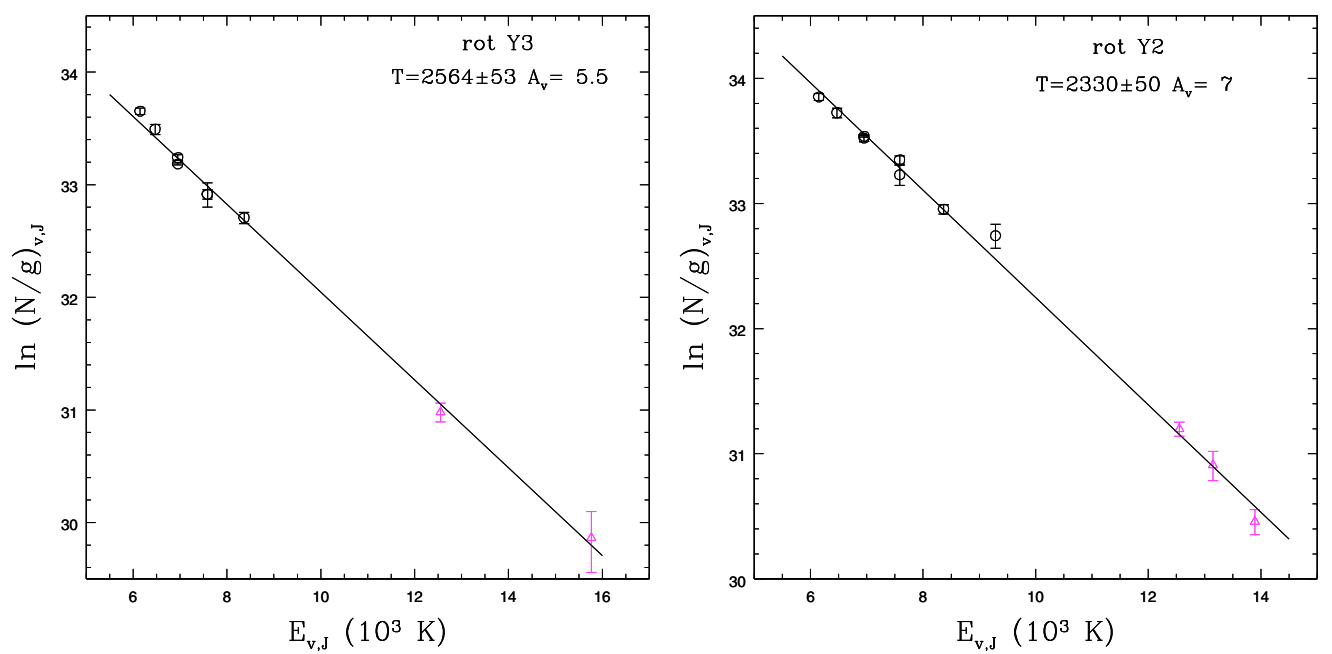

Fig. B.1. As Fig. 6 for knot Y3 and knot Y2.
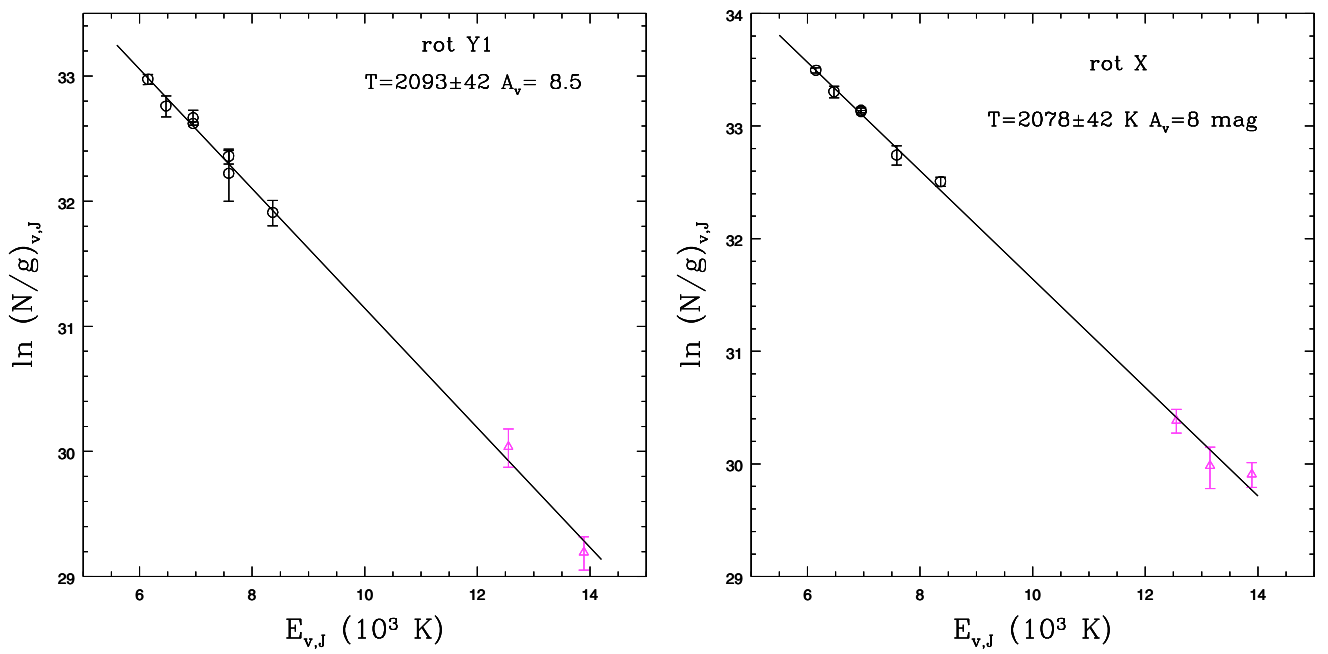

Fig. B.2. As Fig. 6 for knot $Y 1$ and knot $X$. 